\title{
Solution of stress intensity factors for 2-D multiple crack problems by the fast multipole boundary element method
}

\author{
Zhao Guo', Yijun $\mathrm{Liu}^{2} \&$ Hang $\mathrm{Ma}^{3}$ \\ ${ }^{1}$ Shanghai Institute of Applied Mathematics and Mechanics, \\ Shanghai University, P. R. China \\ ${ }^{2}$ Mechanics Engineering, University of Cincinnati, USA \\ ${ }^{3}$ Department of Mechanics, Shanghai University, P. R. China
}

\begin{abstract}
The fast multipole boundary element method (BEM) based on both singular and hypersingular boundary integral equations (BIEs) for 2-D elasticity is applied to study 2-D multiple crack problems in this work. For multiple crack problems, the degrees of freedom (DOFs) and the size of the matrices can increase quickly as the number of cracks increases, which prohibits the use of the conventional BEM. In this study, constant line elements are used to discretize the crack surfaces and other boundaries. There is no use of the special quarter-point elements at crack tips in the present approach. To account for the need for accuracy, a relatively larger number of constant elements is required on the surfaces of cracks. By applying the fast multipole BEM, the increased computational work can be dealt with readily on desktop PCs. Results of the computed stress intensity factors using crack opening displacements (CODs) near the crack tips are presented in this paper. It is found that the fast multipole BEM with constant elements can predict the stress intensity factors of cracks in 2-D plates with sufficient accuracy and efficiency.
\end{abstract}

Keywords: fast multipole boundary element method, 2-D crack problems, constant boundary elements, stress intensity factors.

\section{Introduction}

Engineering materials often contain multiple cracks, such as ceramics, concretes, brittle materials, aircrafts as well as earthquakes. The elastic behavior and 
fracture characteristics of elastic solids with multiple cracks need to be studied, where the present of cracks is a common reason for the failure of materials which has always been a challenge for researchers and engineers. The fundamental postulate of linear elastic fracture mechanics (LEFM) is that the crack behaviors are determined by the value of stress intensity factors (SIFs) at the crack tips, which is a function depends on the applied load, the crack size and the geometry configuration of the cracked plate. Since Irwin first postulated that the crack behavior is determined by the value of SIFs at the crack tips, the computation of SIFs has been playing a very important role in linear elastic fracture mechanics. However, due to their complexity, the computation of crack behaviors has been a challenge for researchers, and many multiple crack problems still have not been solved by using analytical methods.

The boundary element method (BEM) has been considered to be advantageous in solving LEFM problems [1, 2], because of its boundary discretization nature. However, while the BEM has enjoyed the reputation of easy meshing in modeling, the efficiency and computation storage have been a serious concern for analyzing large-scale problems because of its dense and asymmetric coefficient matrix. To reduce the CPU time and memory storage, the fast multipole method (FMM) proposed by Greengard and Rokhlin [3] has been applied to the BEM for more than two decades, leading to the so-called fast multipole BEM. The fast multiple BEM and its applications were developed intensively by many researchers in the last two decades [4, 5].

For multiple crack problems, it is well-known that the size of the matrices can be very large as the number of cracks increases. Because of this, multiple crack problems usually need large BEM models, possibly millions of degrees of freedom (DOFs) in some large-scale cases, which can lead to a time-consuming calculation on desktop PCs. To reduce the CPU time and memory storage, the fast multipole BEM is applied for solving multiple crack problems in the present paper. Firstly, the outer boundary and crack surfaces of the model are discretized in the same way as in conventional BEM. Secondly, a quad-tree structure is constructed. Then the multipole expansion and local expansion of the kernel functions are used and the generalized minimal residual method (GMRES) is applied as the iterative solver to obtain the unknowns on boundaries. The CPU time and memory storage in most cases can be reduced to about $O(N)$, where $N$ is the number of DOFs. From the basic BIE formulations, it is clear that if all the displacements discontinuities for the cracks are known, the multiple crack problems such as computing the stress intensity factor using crack opening displacements near the crack tip obtained from the fast multipole BEM or stress distributions can be solved.

In the following sections, the BIE formulations for crack analysis are briefly reviewed first. Then, the main steps of the fast multipole BEM are briefly summarized. The comparison of crack opening displacements from the fast multipole BEM and ANSYS will be described. Finally, numerical examples of models with large numbers of cracks in finite/infinite plates are simulated to show the accuracy and efficiency of the proposed approach. 


\section{Boundary integral equation for multiple crack problems}

Consider a 2-D elastic solid $\Omega$ with multiple $N_{\mathrm{C}}$ ideal cracks, the displacements at the source point $y$ can be expressed by the conventional boundary integral equation (CBIE) on the outer surface $A_{m}$ in terms of the crack opening displacements (CODs):

$$
\begin{aligned}
\gamma u_{i}(y) & =\int_{\Gamma} \tau_{j}(x) U_{i j}(x, y) d \Gamma(x)-\int_{\Gamma} u_{j}(x) T_{i j}(x, y) d \Gamma(x) \\
& -\sum_{m=1}^{N_{C}} \int_{A_{m}^{+}} \Delta u_{j}(x) T_{i j}(x, y) d A(x), \quad y \in(\Gamma \cup \Omega) \backslash A_{m}\left(m=1, \cdots, N_{C}\right)
\end{aligned}
$$

where $\Gamma$ is the outer boundary of the 2-D solid, and $\gamma=1$ if $y \in \Omega$ and $\gamma=0.5$ if $y \in \Gamma$ when $\Gamma$ is smooth. $U_{i j}(x, y), T_{i j}(x, y)$ respectively represent the Kelvin displacement and traction fundamental solutions. $\Delta u_{i}$ is the components of relative displacement discontinuities between two surfaces of the crack defined by:

$$
\Delta u_{i}(x)=\left.u_{i}(x)\right|_{x \in A^{+}}-\left.u_{i}(x)\right|_{x \in A^{-}}
$$

where $A^{+}$and $A^{-}$represent the upper and the lower surfaces of the crack, respectively. Therefore, the displacement $u_{i}(y)$ can be determined by the traction $\tau_{j}(x)$, the displacement $u_{j}(x)$ and the COD on the crack surfaces. It is clear that if all the CODs are known, the multiple crack problems can be solved in discrete form as the conventional BEM. However, to solve this given multiple crack problems, the solutions of the unknowns are still impossible to obtain by CBIE (1). An additional BIE should be constructed on the opposite crack surface, that is, the traction boundary integral equation (HBIE):

$$
\begin{array}{r}
\gamma \tau_{i}(y)=n_{j}(y) \int_{\Gamma} \tau_{k}(x) U_{i j k}(x, y) d \Gamma(x)-n_{j}(y) \int_{\Gamma} u_{k}(x) T_{i j k}(x, y) d \Gamma(x) \\
-n_{j}(y) \sum_{m=1}^{N_{C}} \int_{A_{m}^{+}} \Delta u_{k}(x) T_{i j k}(x, y) d A(x), y \in(\Gamma \cup \Omega) \backslash\left(\cup A_{m}\right)
\end{array}
$$

where $n_{j}$ denotes the component of the outward unit normal at the source point $y$. The kernel functions $U_{i j k}(x, y)$ and $T_{i j k}(x, y)$ contains the derivatives of $U_{i j}(x, y)$ and $T_{i j}(x, y)$ together with elastic constants.

It is worthwhile to note that, the kernel functions will exhibit a singularity as the source and field points are coincided. In the present paper, a CHBIE formulation using a linear combination of the CBIE and HBIE can be written as [4]:

$$
\mathrm{CBIE}+\beta \mathrm{HBIE}=0
$$

where $\beta$ is the coupling constant. 
Once the entire unknown CODs on the crack surfaces are obtained by applying the fast multipole BEM, the SIFs at the two crack tips can easily be obtained by using one point formula as:

$$
K_{I}=\frac{G}{(\kappa+1)} \sqrt{\frac{2 \pi}{r}} \Delta u_{n}(r), K_{I I}=\frac{G}{(\kappa+1)} \sqrt{\frac{2 \pi}{r}} \Delta u_{s}(r)
$$

where $\mathrm{G}$ stands for the shear modulus and $r$ is the distance from the observed point to the crack tip. $\Delta u_{n}(r)$ and $\Delta u_{s}(r)$ are the opening and sliding displacements at the observed point. The parameter $\kappa$ is defined as:

$$
\kappa= \begin{cases}3-4 v & \text { for plane strain problem } \mathrm{s} \\ \frac{3-v}{1+v} & \text { for plane stress problem } \mathrm{s}\end{cases}
$$

where $v$ stands for the Poisson's ratio.

\section{Formulation of the Fast multipole BEM}

The fast multipole BEM has been investigated by many authors for more than two decades. Applications of fast multipole BEM can be found in [4] for solving 2-D and 3-D potential, elastostatic, Stokes flow and acoustic wave problems. A comprehensive review of the fast multipole BEM was given by Nishimura in [5]. For multiple crack problems, Wang and Yao $[6,7]$ have applied a fast multipole BEM using a new form of complex Taylor series expansions and expressive results were obtained using higher-order elements for 2-D problems. Propagations of multiple cracks were also studied by Wang and Yao [6, 7] based on the SIFs approach.

In this section, the complex notation of the kernel functions, expansions and corresponding translations in the FMM are summarily derived for the multiple crack problems. However, since the main multipole steps of $U_{i j}(x, y)$ and $T_{i j}(x, y)$ kernel integral are similar. We do not expand the relatively complicated $T_{i j}(x, y), U_{i j k}(x, y)$ and $T_{i j k}(x, y)$ directly but expand the kernel function $U_{i j}(x, y)$ only. The representations of kernel functions in HBIE (3) can also be found in [4].

\subsection{Complex representations of the kernels}

Define the complex number $z_{0}$ and $z$ as: $z_{0}=y_{1}+i y_{2}$ and $z=x_{1}+i x_{2}$, where $x_{1}$ and $x_{2}$ are the ordinates of $x, y_{1}$ and $y_{2}$ are the ordinates of $y$ as shown in Fig. 1. By using the complex notation [4] the expansion of $U$ kernel function can be written in the following complex form:

$$
\frac{1}{2} u\left(z_{0}\right)=D_{t}\left(z_{0}\right)-D_{u}\left(z_{0}\right)
$$


where $u=u_{1}+i u_{2}$ is the complex representation of the displacement, and we have:

$$
\begin{aligned}
D_{t}\left(z_{0}\right)=\frac{1}{2 \mu(1+\kappa)} \int_{s}[ & {\left[\kappa G\left(z_{0}, z\right) t(z)-\left(z_{0}-z\right) \overline{G^{\prime}\left(z_{0}, z\right)} \overline{t(z)}\right.} \\
& \left.+\kappa \overline{G\left(z_{0}, z\right) t}(z)\right] d S(z) \\
D_{u}\left(z_{0}\right)=- & \frac{1}{1+\kappa} \int_{s}\left\{\kappa G^{\prime}\left(z_{0}, z\right) n(z) u(z)-\left(z_{0}-z\right) \overline{G^{\prime \prime}\left(z_{0}, z\right)} \overline{n(z)} \overline{u(z)}\right. \\
& \left.+\overline{G^{\prime}\left(z_{0}, z\right)}[n(z) \overline{u(z)}+\overline{n(z) u}(z)]\right\} d S(z)
\end{aligned}
$$

where the over bar indicates complex conjugate and $G$ is the Green's function (in complex form) for 2-D potential problems which is given by:

$$
G\left(z_{0}, z\right)=-\frac{1}{2 \pi} \log \left(z_{0}-z\right)
$$

where ()$^{\prime}=\partial() / \partial z_{0}$. Eq. (8) represents the complex notation of $U$ kernel and Eq. (9) represents the complex notation of $T$ kernel in CBIE (1), respectively.

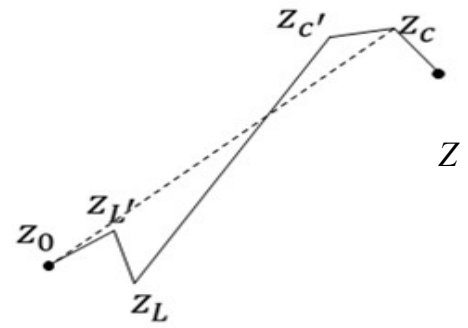

Figure 1: Complex notation for fast multipole expansions.

Let $z_{c}$ be a multipole expansion point close to $z\left(\left|z-z_{c}\right| \ll\left|z_{0}-z_{c}\right|\right)$ and apply Taylor series expansion, the multipole expansion of $G\left(z_{0}, z\right)$ and its derivatives are:

$$
\begin{aligned}
& G\left(z_{0}, z\right)=-\frac{1}{2 \pi} \log \left(z_{0}-z\right)=\frac{1}{2 \pi} \sum_{k=0}^{\infty} O_{k}\left(z_{0}-z_{c}\right) I_{k}\left(z-z_{c}\right) \\
& \frac{\partial^{(l)} G\left(z_{0}, z\right)}{\partial z_{0}^{(l)}}=(-1)^{l} \frac{1}{2 \pi} \sum_{k=0}^{\infty} O_{k+l}\left(z_{0}-z_{c}\right) I_{k}\left(z-z_{c}\right) \quad \text { for } \quad l=1,2, \ldots,
\end{aligned}
$$

where the two auxiliary functions are defined as: 


$$
\begin{gathered}
I_{k}(z)=\frac{z^{k}}{k !}, \text { for } k \geq 0 \\
O_{0}(z)=-\log (z) \\
O_{k}(z)=\frac{(k-1) !}{z^{k}}, \quad \text { for } k \geq 1
\end{gathered}
$$

\subsection{Multipole expansion and moment}

In the multipole expansion, the boundary integral of kernel functions is expanded into a series of products of functions. The multipole expansion for Eq. (8) is given by:

$$
\begin{gathered}
D_{t}\left(z_{0}\right)=\frac{1}{4 \pi \mu(1+\kappa)}\left[\kappa \sum_{k=0}^{\infty} O_{k}\left(z_{0}-z_{c}\right) M_{k}\left(z_{c}\right)+z_{0} \sum_{k=0}^{\infty} \overline{O_{k+1}\left(z_{0}-z_{c}\right)} \overline{M_{k}\left(z_{c}\right)}\right. \\
\left.+\sum_{k=0}^{\infty} \overline{O_{k}\left(z_{0}-z_{c}\right)} N_{k}\left(z_{c}\right)\right]
\end{gathered}
$$

where the two moments are defined by:

$$
\begin{aligned}
& M_{k}\left(z_{c}\right)=\int_{S_{c}} I_{k}\left(z-z_{c}\right) t(z) d S(z), \quad \text { for } k \geq 0 \\
& N_{0}=\kappa \int_{S_{c}} t(z) d S(z) \\
& N_{k}\left(z_{c}\right)=\int_{S_{c}}\left[\kappa \overline{I_{k}\left(z-z_{c}\right) t}(z)-\overline{I_{k-1}\left(z-z_{c}\right)} \overline{t(z)}\right] d S(z)
\end{aligned}
$$

where $S_{c}$ is a subset of $S$ which is far away from the source point $z_{0}$.

\subsection{Moment-to-moment translation}

If the multipole expansion point shifts to $z_{c}$ from $z_{c}$, then the moment to moment translation (M2M) can be obtained, as follows:

$$
M_{k}\left(z_{c^{\prime}}\right)=\sum_{l=0}^{k} I_{k-l}\left(z_{c}-z_{c^{\prime}}\right) M_{l}\left(z_{c}\right), \text { for } k \geq 0
$$

Similarly, we have:

$$
N_{k}\left(z_{c^{\prime}}\right)=\sum_{l=0}^{k} \overline{I_{k-l}\left(z_{c}-z_{c^{\prime}}\right)} N_{l}\left(z_{c}\right), \quad \text { for } k \geq 0
$$




\subsection{Local expansion and moment-to-local translation}

If the source point $z_{0}$ is moved to $z_{L}$, that is $\left|z_{0}-z_{L}\right| \ll\left|z_{c}-z_{L}\right|$. By using a Taylor series expansion, the following local expansion can be obtained:

$$
\begin{aligned}
D_{t}\left(z_{0}\right)=\frac{1}{4 \pi \mu(1+\kappa)}[ & \kappa \sum_{l=0}^{\infty} I_{l}\left(z_{0}-z_{L}\right) L_{l}\left(z_{L}\right)-z_{0} \sum_{l=0}^{\infty} \overline{I_{l-1}\left(z_{0}-z_{L}\right)} \overline{L_{l}\left(z_{L}\right)} \\
& \left.+\sum_{l=0}^{\infty} \overline{I_{l}\left(z_{0}-z_{L}\right)} K_{l}\left(z_{L}\right)\right]
\end{aligned}
$$

where the two coefficients are given by:

$$
\begin{gathered}
L_{l}\left(z_{L}\right)=(-1)^{l} \sum_{k=0}^{\infty} O_{k+l}\left(z_{L}-z_{c}\right) M_{k}\left(z_{c}\right), \quad \text { for } l \geq 0 ; \\
K_{l}\left(z_{L}\right)=(-1)^{l} \sum_{k=0}^{\infty} \overline{O_{k+l}\left(z_{L}-z_{c}\right)} N_{k}\left(z_{c}\right), \text { for } l \geq 0
\end{gathered}
$$

\subsection{Local-to-local translation}

If the local expansion center in Eq. (18) is moved to $z_{L^{\prime}}$ from $z_{L}$, then the local to local translations (L2L) can be obtained, as follows:

$$
\begin{aligned}
& L_{l}\left(z_{L^{\prime}}\right)=\sum_{m=l}^{\infty} I_{m-l}\left(z_{L^{\prime}}-z_{L}\right) L_{m}\left(z_{L}\right), \text { for } l \geq 0 ; \\
& K_{l}\left(z_{L^{\prime}}\right)=\sum_{m=l}^{\infty} \overline{I_{m-l}\left(z_{L^{\prime}}-z_{L}\right)} K_{m}\left(z_{L}\right), \text { for } l \geq 0
\end{aligned}
$$

\section{Two main steps of the fast multipole model}

In this section, we repeat to simply discuss the two important procedures of fast multipole BEM, i.e., the construction of quad-tree and the integration of kernel functions. The fast multipole BEM used the same discretization as the conventional BEM. The boundaries of the domain and the internal domain are discretized using constant elements, respectively. Consider a minimum red square that covers the internal domain which is defined as level 0. Correspondingly, the internal domain is defined as cell $\mathrm{c} 1$. Then start to divide the red square into four equal yellow cells of level 1. Continue to divide the cell in this way until each small cell contains one node. If a cell does not contain any node, there is no need to do the above dividing process. The structure of discretization is illustrated in details as shown in Fig. 2.

After the upward pass and downward pass are finished, the integration of kernel functions will be evaluation as shown in Fig. 3. 

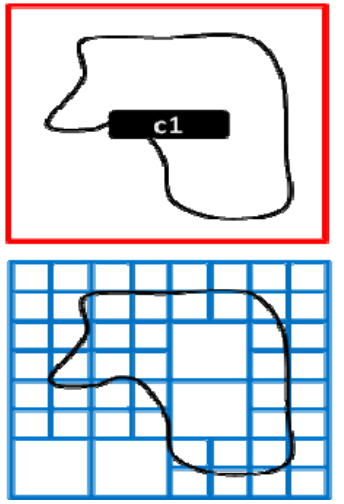
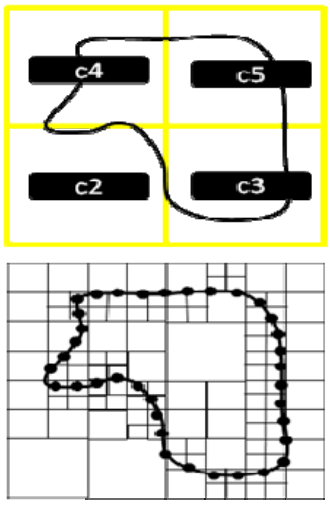
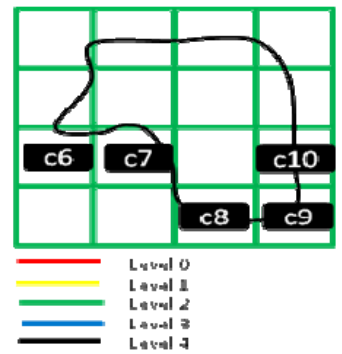

Figure 2: Quad-tree structure of the boundary element mesh.

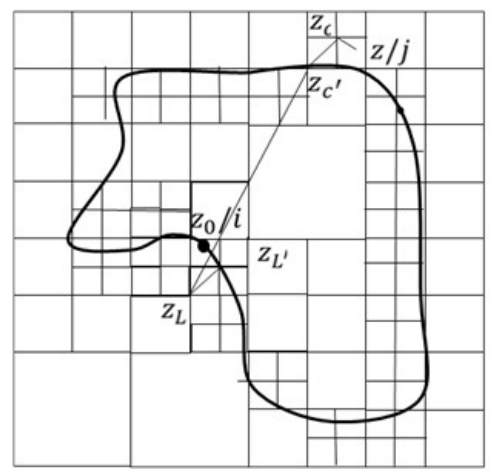

Integral of kemels of element $i$ :

Get $M$ of the cell from element $j$;

Compute $M$ for cell of $j$ at level 2 by M2M;

Compute $L$ for cell of $j$ at level 2 by $\mathrm{M} 2 \mathrm{~L}$;

Compute $L$ for element $i$ by L2L;

Get integral by local expansion.

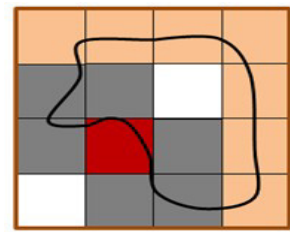

cell level 3

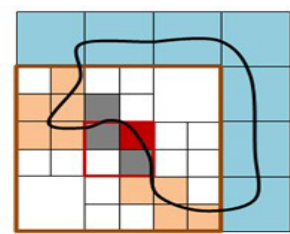

cell level 4

- Compute moments for all cells

- Start from level 2

$\checkmark$ Get $L$ for target cell by M2L of cells in interaction list

- Go to lower level

$\checkmark$ Get $L$ for target cell by M2L of cells in interaction list

$\checkmark$ Get $L$ for target cell from its parent

- Reach leaf

$\checkmark$ Get $L$ for element, compute integral by local expansion

$\checkmark$ Direct compute integral from adjacent cells

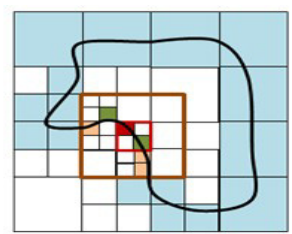

cell level 5 - leaf

Cell in interaction list

Far cells

Adjacent cells

Target cell

Parent of target cell

None cell(no element)

Figure 3: Integrals of the kernel functions.

\section{Numerical examples}

In the numerical examples, the outer boundaries and crack surfaces are discretized using constant elements. The number of terms in multipole expansion 
and local expansion are set at 20. The tolerance for convergence using GMRES in the present examples is chosen as $10^{-6}$. For the numerical examples, only $\mathrm{K}_{\mathrm{I}}$ is computed. The computation of $\mathrm{K}_{\mathrm{II}}$ is easily obtained as well.

\subsection{Comparison of the COD results between the BEM and ANSYS}

The first example is constructed for the purpose of illustrating the computational procedure presented. Without loss of generality, consider the computational model contains a single center crack $\left(N_{C}=1\right)$ in a 2-D square plate as shown in Fig. 4. For the outer boundary length $2 W=20 \mathrm{~mm}$, the crack length $2 a=2 \mathrm{~mm}$. The material properties are $E=1 \mathrm{Mpa}$ and Poisson's ratio $v=0.3$, respectively. The stresses at upper and lower boundary are $\sigma=1 \mathrm{Mpa}$.

This example was analyzed using the present fast multipole BEM and ANSYS software. In the ANSYS analysis, the crack was divided into 90 constant elements while 50 constant elements for each outer boundary. Fig. 5 compares the COD results on the crack surface predicted by the two numerical

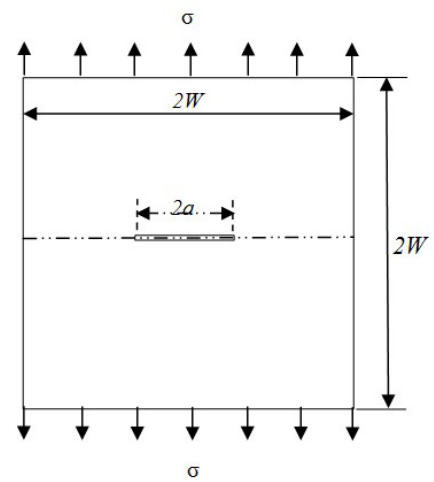

Figure 4: $\quad$ Center crack in a square plate.

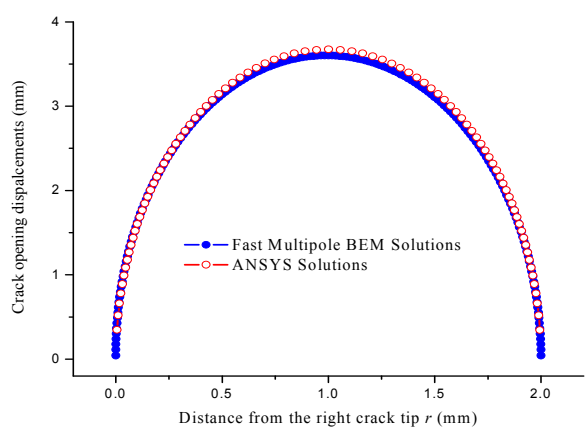

Figure 5: COD comparison from the fast multipole BEM and ANSYS. 
analyses. The fast multipole BEM results agree well with ANSYS solutions. The maximum error of the fast multipole BEM results is less than $2.0 \%$.

\subsection{A plate with two symmetrically inclined cracks}

The second example is a finite plate in tension with two symmetrically inclined cracks of equal size as shown in Fig. 6. In the present model, the outer boundaries are discretized by using 400 constant elements, while each crack surface is discretized by using 360 constant elements.

The results of the normalized mode-I stress intensity factors as a function of the inclined angle $\theta$ at the crack tip A and B are present as shown in Fig. 7, respectively. It can be known that the computed results of the proposed approach are quite agreed with Chen and Chong [8]. The accuracy has been greatly improved compared with the author's previous work [9] as well.

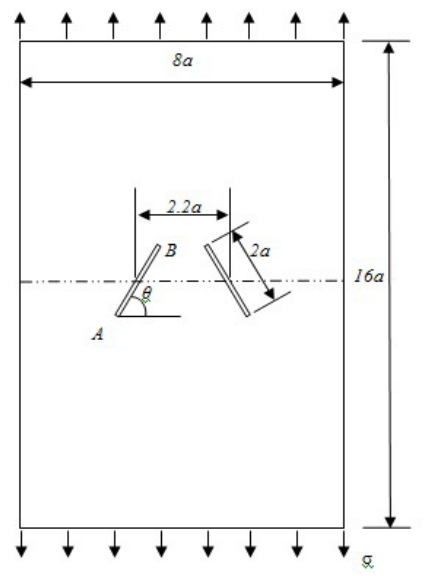

Figure 6: A plate in tension with two symmetrically inclined cracks.

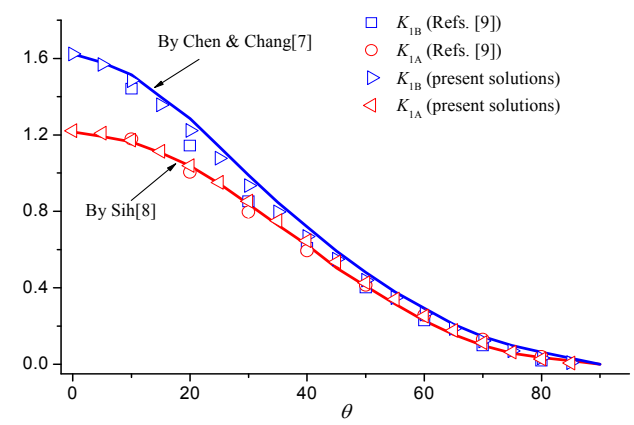

Figure 7: $\quad$ Normalized mode-I SIFs as a function of the tilting angle $\theta$. 


\subsection{An infinite plate with two collinear cracks}

The third example is an infinite plate in tension which contains a pair of collinear cracks with equal length. The computed results of the normalized mode-I stress intensity factors for this example are presents as shown in Fig. 8. It can be seen that the numerical results from the proposed approach are in good agreement with the analytical solutions of Ref. [10].

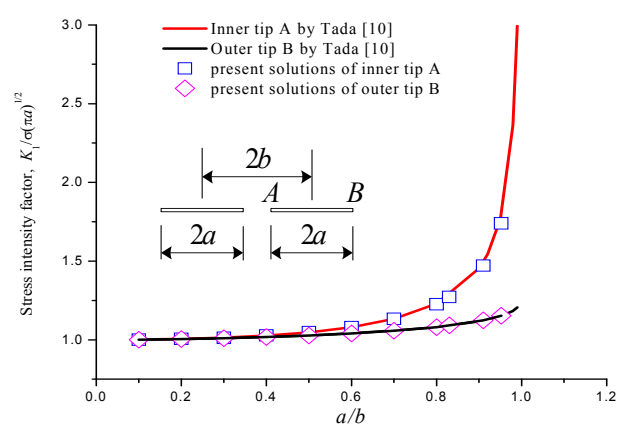

Figure 8: Normalized mode-I SIFs as a function of $a / b$ for two collinear cracks.

\subsection{One row of periodical collinear cracks}

The last example presents a row of periodical collinear cracks in an infinite plate with the same size, orientation and spacing under a far-field tension perpendicular to the crack faces. In the computation, up to 2001 cracks are taken into consideration instead of using an infinite number of cracks. The purpose of this example is to compute the normalized mode-I SIFs. On the other hand, yet is also the most important point is to find an appropriate number of cracks to represent the infinite cases. The normalized mode-I stress intensity factors are calculated by the present approach and Ref. [10], compared as shown in Fig. 9.

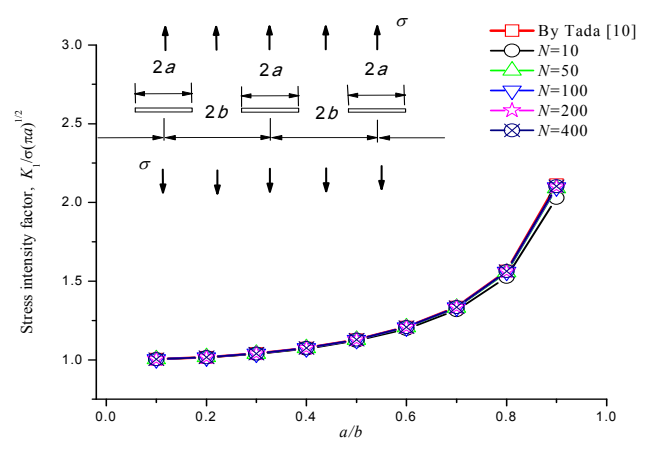

Figure 9: $\quad$ Normalized mode-I SIFs for periodical collinear equal cracks. 
It shows a good agreement between the numerical and the analytical solutions when $N=10,50,100,200,400$. It also can be seen that the numerical results are gradually closed to the analytical solutions when the number of cracks increasing. The absolute errors of SIFs prove this argument as shown in Fig. 10 with different $N$, respectively. In general, the increase of the number of cracks can improve the accuracy of the present results. Correspondingly, both CPU time and the memory requirement will tend to long and large, respectively. At this point, a finite number of cracks can stand for this class of multiple crack problems.

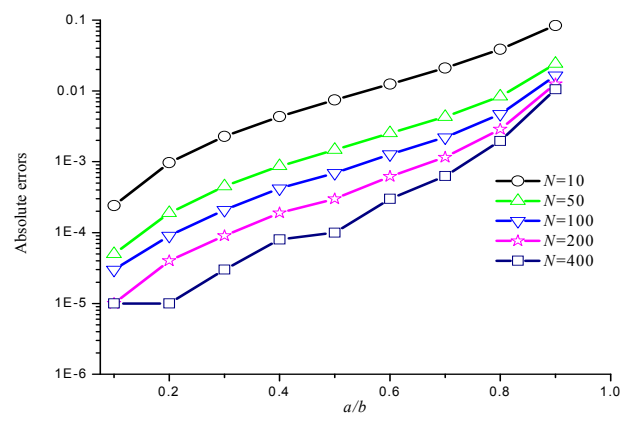

Figure 10: The absolute errors of SIFs as a function of $a / b$ using different number of $N$.

\section{Numerical examples}

A fast multipole BEM was applied to 2-D multiple crack analysis. Constant elements were used on both the outer boundaries and crack surfaces. There is no use of any special crack-tip elements in crack tip discretization in the fast multipole BEM as well as ANSYS analysis. The effectiveness and the accuracy of the proposed approach were verified by computing the normalized mode-I stress intensity factors in some examples. The CPU times take within minutes, and the required memory is less compared with conventional BEM. Numerical examples show that the approach is accurate and efficient.

Further work will focus on the propagation of multiple cracks. The 2-D work with the fast multipole BEM needs to be extended to 3-D cases. Other fast solution methods for the BEM, such as the adaptive cross approximation (ACA) method, can also be applied to study crack related problems.

\section{Acknowledgements}

The first author Zhao Guo would like to gratefully acknowledge the financial support by grant 11272195 of the National Natural Science Foundation of China, the fellowship of the China Scholarship Council (CSC). Zhao Guo would also like to thank Prof. Y. J. Liu, Mr. Shuo Huang and Miss. Anli Wang at the 
University of Cincinnati for their help in the multiple crack problems modeling and programming.

\section{References}

[1] Brebbia, C. A., Telles, J. C. F. and Wrobel, L. C., Boundary element techniques [M]. Berlin: Springer-Verlag, 1984.

[2] Aliabadi, M. H., Boundary element formulations in fracture mechanics [J]. Applied Mechanics Reviews, 1997, 50(2), 83-96.

[3] Greengard, L. F. and Rokhlin, V., A fast algorithm for particle simulations [J]. J Comput Phys, 1987, 73, 325-48.

[4] Liu, Y. J., Fast multipole boundary element method - theory and applications in engineering [M]. London: Cambridge University Press; 2009.

[5] Nishimura, N., Fast multipole accelerated boundary integral equation methods [J]. Applied Mechanics Review, 2002, 55(4), 299-324.

[6] Wang, P. B. and Yao, Z. H., Fast multipole DBEM analysis of fatigue crack growth [J]. Comput. Mech., 2006, 38, 223-233

[7] Wang, P. B. and Yao, Z. H., Fast multipole BEM for simulation of 2-D solids containing large numbers of cracks $[\mathrm{J}]$. J Tsinghua Science and Technology, 2005, 10(1), 76-81.

[8] Chen, W. H. and Chang, C. S., Analysis of two-dimensional mixed-crack problems for finite element alternating method [J]. Computers and Structures, 1989, 6, 1451-1458.

[9] Guo, Z. and Ma, H., Solution of stress intensity factors of multiple cracks in plane elasticity with eigen COD formulation of boundary integral equation [J]. J Shanghai Univ., 2011, 15(3), 173-9.

[10] Tada, H., Paris, P. C. and Irwin, G. R., The Stress Analysis of Cracks Handbook [M], 1973. 